\title{
Visible light photocatalysis of methylene blue using cobalt substituted cubic $\mathrm{NiO}$ nanoparticles
}

\author{
AMITA KHATRI and PAWAN S RANA* \\ Department of Physics, D.C.R. University of Science and Technology, Murthal 131039, India \\ *Author for correspondence (drpawansrana.phy@dcrustm.org)
}

MS received 18 August 2018; accepted 17 December 2018; published online 4 May 2019

\begin{abstract}
This study reports the facile co-precipitation synthesis of pure and cobalt substituted NiO nanoparticles. The cubic crystalline structure of the synthesized $\mathrm{NiO}$ nanoparticles was confirmed through X-ray diffraction. Structural behaviour, morphological, elemental composition and optical properties of the prepared nanoparticles are investigated by Fourier transform infrared spectroscopy, scanning electron microscopy-energy dispersive spectrum, transmission electron microscopy and UV-Vis spectroscopy. The optical band gap is determined using UV spectra and is observed to decrease with an increase in cobalt ion concentration. Furthermore, methylene blue dye solution has been used to investigate the photocatalytic ability of the synthesized nanoparticles in the presence of sunlight. It is found that percentage degradation in dye concentration increases with irradiation time and also with the increase in the concentration of doping of cobalt in NiO. The dye solution is found to degrade to $94 \%$ within $50 \mathrm{~min}$ in the presence of solar radiation and the $\mathrm{Ni}_{0.6} \mathrm{Co}_{0.4} \mathrm{O}$ sample.
\end{abstract}

Keywords. NiO; UV-Vis spectroscopy; photocatalysis.

\section{Introduction}

The advances in technology and industrialization impose a global threat to human life and the environment. Good quality of water is a precious resource which is necessary for sustaining all forms of life but the number of factors has led to pollution of water. Organic dyes disposed from textile industries constitute a major water pollutant. The release of this coloured wastewater in the environment through a chemical reaction taking place can produce harmful byproducts which may be eutrophic. The polluted water needs to be treated in order to improve its quality which has led to a number of studies being carried out by researchers in this regard. The colour removal has been extensively studied using different methods such as coagulation [1], ultra-filtration [2], nanofiltration, flocculation, reverse osmosis, activated carbon [3], etc. However, these methods are inefficient having operational problems such as generation of sludge, which leads to disposal problems and require further separation techniques adding more cost to the entire process. An alternative to this, an advanced oxidation process (AOP) is used which starts with the generation of strong hydroxyl radicals, which can completely degrade the pollutant into less toxic or harmless products $\left(\mathrm{CO}_{2}\right.$ and $\left.\mathrm{H}_{2} \mathrm{O}\right)$ [4-6]. The main supremacy of the AOP is that there is no consumption of chemicals, no sludge production and no strict lab conditions are required [7]. The widely used AOP includes photolysis, fenton method, photofenton, ozonolysis, sonolysis and photocatalysis [8]. Out of these AOP processes, photocatalysis uses visible and ultraviolet (UV) radiation from sunlight and in the presence of suitable catalysts degrades the organic pollutants present in wastewater through a chemical reaction.

During the past decade, metal oxide nanoparticles have been studied as efficient photocatalysts and have been used to degrade synthetic dyes under different conditions $[9,10]$. The main features to be effective photocatalysts are stability, non-toxicity, suitable morphology, appropriate band gap and reusability [10]. Nickel oxide (NiO) nanoparticles have been effectively used as photocatalysts [4]. $\mathrm{NiO}$ is a stable p-type semiconductor metal oxide having tendency to absorb UV radiations due to its wide band gap and therefore a small portion of the solar spectrum is absorbed by this material [11]. NiO nanoparticles find potential applications in gas sensors [12-15], fuel cells [16], electrochromic films [17,18] and heterogeneous catalytic materials. To date, different methods have been employed to synthesize $\mathrm{NiO}$ nanoparticles, namely, thermal decomposition [19], hydrothermal [20], pulsed-laser ablation [21], solvothermal [22], anodic arc plasma method [23], sol-gel [24] and chemical co-precipitation methods [25]. The synthesis route and conditions may affect various properties of these nanoparticles. In addition to the synthesis route, doping with other metal ions has also been employed to improve the physicochemical properties of nanomaterials which is essential for their various applications [26].

Motahari et al [27] synthesized NiO nanostructures by the thermal decomposition method for the photocatalyst application and reported that $\mathrm{NiO}$ nanoparticles act as highly efficient photocatalysts towards degradation of Rhodamine B under UV radiation. Gnanasekaran et al [28] reported the synthesis of metal oxide nanoparticles $\left(\mathrm{CeO}_{2}, \mathrm{NiO}, \mathrm{ZnO}\right.$, etc. $)$ 
by the precipitation method and degradation of textile dyes using these metal oxide nanoparticles. In the present work, firstly $\mathrm{Ni}_{1-x} \mathrm{Co}_{x} \mathrm{O}(x=0,0.1,0.2$ and 0.4$)$ cubic nanostructures were synthesized by the chemical co-precipitation method which is simpler as compared to other methods and is a facile method of synthesis without requiring any expensive equipment. Surface morphologies and structural features of the samples were characterized by X-ray powder diffraction (XRD), Fourier transform infrared (FTIR) spectroscopy, ultraviolet-visible (UV-Vis) spectroscopy, scanning electron microscopy-energy dispersive X-ray spectroscopy (SEMEDS) and transmission electron microscopy (TEM). These nanostructures were then utilized to degrade methylene blue (MB) dye through photocatalysis in the presence of sunlight.

\section{Experimental}

\subsection{Materials}

All the chemicals and reagents used in our work were purchased from Merck and were used as received without further purification. Double distilled water was used throughout the synthesis process. The reagents used were analar grade nickel chloride hexahydrate $\left(\mathrm{NiCl}_{2} \cdot 6 \mathrm{H}_{2} \mathrm{O}\right)$, cobalt(II) chloride hexahydrate $\left(\mathrm{CoCl}_{2} \cdot 6 \mathrm{H}_{2} \mathrm{O}\right), \mathrm{NaOH}$ flakes and $\mathrm{MB}$ dye (Fisher Scientific).

\subsection{Synthesis of nanoparticles}

$\mathrm{Ni}_{1-x} \mathrm{Co}_{x} \mathrm{O}(x=0,0.1,0.2$ and 0.4$)$ nanoparticles were synthesized by the chemical precipitation method [29]. In this procedure, homogeneous solutions of the required amount of $\mathrm{NiCl}_{2} \cdot 6 \mathrm{H}_{2} \mathrm{O}$ and $\mathrm{CoCl}_{2} \cdot 6 \mathrm{H}_{2} \mathrm{O}$ were prepared by dissolving them in double distilled water using a magnetic stirrer. Both of these solutions were then mixed using a stirrer and the $\mathrm{pH}$ of the solution was increased through dropwise addition of $\mathrm{NaOH}$ with continuous stirring for $30 \mathrm{~min}$. The light green precipitates obtained were then aged under ambient conditions for $24 \mathrm{~h}$. Then, the resulting green precipitates were centrifuged for $10 \mathrm{~min}$ at $4000 \mathrm{rpm}$ and washed several times with distilled water to remove the impurities and then dried overnight at $70^{\circ} \mathrm{C}$. Finally, the obtained products were pulverized using a mortar-pestle and annealed at $400^{\circ} \mathrm{C}$ for $4 \mathrm{~h}$ to obtain the desired nanoparticles. The prepared samples were named as pure and cobalt substituted $\mathrm{NiO}$ nanoparticles.

\subsection{Characterization}

XRD was carried out on an X-ray diffractometer (Rigaku Ultima-IV) for the determination of the crystalline nature of the prepared samples. Spectra were recorded in the $2 \theta$ range of $20-80^{\circ}$ with a scanning speed of $2^{\circ} \mathrm{min}^{-1}$ using $\mathrm{CuK} \alpha$ radiation $(\lambda=1.54 \AA$ ) as the X-ray source. Room temperature FTIR spectra were recorded on an FTIR spectrophotometer
(Perkin Elmer Frontier) in the wavenumber range of 4000$400 \mathrm{~cm}^{-1}$. The standard $\mathrm{KBr}$ pellet technique was employed to carry out IR measurements [30]. Morphological and elemental composition analyses of all samples were carried out on a scanning electron microscope-energy dispersive spectrometer (SIGMA-ZEISS SIGMA 5.05) at an acceleration voltage of $5 \mathrm{kV}$. Transmission electron microscopy (TECHNAI) at an acceleration voltage of $200 \mathrm{kV}$ was performed to gain an insight into the morphology of nanoparticles. The optical behaviour was investigated by recording UV-Vis spectra on a UV-Vis spectrophotometer (Shimadzu UV-2450). Then the band gap energy of the prepared nanoparticles was derived from the absorbance spectra.

\subsection{Photocatalytic activity}

The photocatalytic activity of the prepared pure and cobalt substituted nickel oxide nanoparticles was assessed by degradation of aqueous solution of $\mathrm{MB}$ dye under sunlight. $\mathrm{MB}$ dye solution with 5 ppm concentration was prepared using double distilled water. One milligram of prepared sample $\mathrm{Ni}_{1-x} \mathrm{Co}_{x} \mathrm{O}$ was added to $100 \mathrm{ml}$ of $\mathrm{MB}$ dye solution. The solution was made basic by increasing the $\mathrm{pH}$ through dropwise addition of $\mathrm{NaOH}$. Before switching to irradiation, the mixture was stirred in dark for $30 \mathrm{~min}$ in order to maintain the adsorption-desorption equilibrium between the catalyst and aqueous $\mathrm{MB}$ dye solution. The dye solution containing catalyst was then exposed to sunlight under continuous stirring. Aliquots were collected at a regular time interval and were analysed to determine the residual amount of MB dye after irradiation by using a UV-Vis spectrophotometer. The percentage degradation was calculated by using the following formula [31]:

$$
\% \text { Degradation }=\frac{\left(A_{0}-A_{\mathrm{t}}\right)}{A_{0}} \times 100,
$$

where $A_{0}$ is the initial absorbance of the MB solution which reached absorbency balance and $A_{\mathrm{t}}$ is the absorbance of the dye solution at irradiation time $(t)$.

\section{Results and discussion}

\subsection{X-ray diffraction}

The room temperature XRD profiles of the synthesized pure and co-substituted $\mathrm{NiO}$ nanoparticles are shown in figure 1. As can be seen, each plot exhibits peaks at $\sim 37.26,43.26$, $62.86,75.44$ and $79.42^{\circ}$ which can be perfectly indexed to (111), (200), (220), (311) and (222) crystal planes of $\mathrm{NiO}$ respectively (JCPDS card no. 47-1049). The diffraction pattern indicates the formation of the FCC structure of pure $\mathrm{NiO}$ and co-substituted $\mathrm{NiO}$ nanoparticles [32]. The relative intensity ordering of each peak also resembles with the standard XRD profile (JCPDS card no. 47-1049) of the 


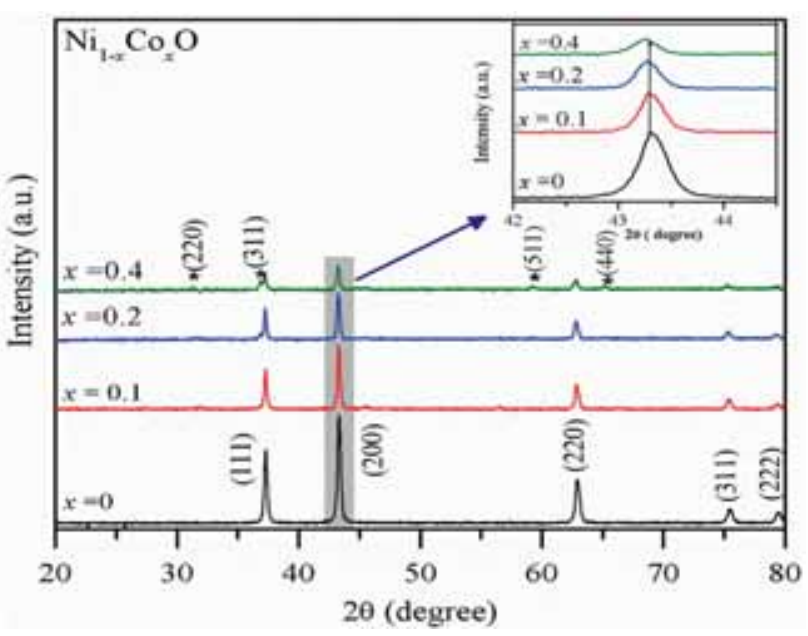

Figure 1. XRD pattern of $\mathrm{Ni}_{1-x} \mathrm{Co}_{x} \mathrm{O}$ nanoparticles.

cubic NiO structure. The intensity and sharpness of peaks mark good crystallinity of the synthesized composition and a little broadening in peaks indicates that particles are in the nanometre range. The broadening of XRD peaks provides a suitable method to measure the average crystallite size. The highest intense peak (200) is considered to find the average crystallite size, $D$ using the Debye-Scherrer formula [33]:

$$
D=\frac{k \lambda}{\beta \cos \theta} .
$$

Here $k$ is the Debye constant equals to $0.9, \lambda$ is the wavelength of $\mathrm{Cu}-\mathrm{K} \alpha$ radiation, $\beta$ is the full-width at half-maximum intensity lines in radians and $\theta$ is the Bragg's [49] angle of the highly intense peak. The estimated values of the crystallite size are reported in table 1 . The lattice parameter ' $a$ ' was calculated from the relation:

$$
d^{2}=\frac{a^{2}}{\left(h^{2}+k^{2}+l^{2}\right)} .
$$

Here $d$ is the interplanar spacing, while $h, k$ and $l$ are the miller indices. The obtained values for the lattice constant are also presented in table 1 . No peak of cobalt is observed at lower content of cobalt in the $\mathrm{NiO}$ lattice indicating that $\mathrm{Co}^{2+}$ ions are incorporated into the lattice site of $\mathrm{Ni}^{2+}$ ions in the $\mathrm{NiO}$ matrix. As the ionic radii of $\mathrm{Ni}^{2+}(0.55 \AA)$ and $\mathrm{Co}^{2+}(0.58 \AA)$ are close to each other, therefore $\mathrm{Co}^{2+}$ can enter the lattice sites of $\mathrm{Ni}^{2+}$ via substitutional doping [34]. A small shift towards the lower angle (see the inset in figure 1) is observed with the enhanced substitution of cobalt, which might be due to occupancy of $\mathrm{Co}^{2+}$ ions at $\mathrm{Ni}$ sites leading to an increase in the lattice constant in $\mathrm{NiO}$ nanoparticles. As evident from table 1 , the average crystallite size of pure $\mathrm{NiO}$ nanoparticles is $24 \mathrm{~nm}$ which is observed to increase with an increase in the concentration of cobalt. A small difference in the ionic radii of $\mathrm{Ni}^{2+}$ and $\mathrm{Co}^{2+}$ leads to an increase in the size of the lattice of substituted $\mathrm{NiO}$ nanoparticles and hence causing a microstructural disorderness [35]. With the addition of cobalt ions, the peak intensity decreases which might be due to an increase in microstrain caused by disorder in the crystalline structure. At higher concentration of cobalt, peaks corresponding to cobalt oxide appear (marked asterisk) at $\sim 31.32,36.88,59.26$ and $65.16^{\circ}$ which are indexed to (220), (311), (511) and (440) crystal planes respectively (JCPDS card no. 01-074-2120) forming composites of $\mathrm{NiO}$ and $\mathrm{Co}_{3} \mathrm{O}_{4}[36,37]$.

\subsection{FTIR spectroscopy}

FTIR spectroscopy is an essential tool to study the functional groups present in the synthesized sample. The infrared vibrations of one component are independent of the presence of other components. The FTIR spectra of all prepared $\mathrm{Ni}_{1-x} \mathrm{Co}_{x} \mathrm{O}(x=0,0.1,0.2$ and 0.4$)$ are depicted in figure 2. As can be seen, all compositions exhibit strong and weak absorption bands/shoulders at $\sim 3846, \sim 3736$, $\sim 3445, \sim 2923, \sim 2854, \sim 2361, \sim 1737, \sim 1638, \sim 1453$, $\sim 1386, \sim 1217, \sim 1162, \sim 1114, \sim 1034, \sim 667, \sim 558$ and $\sim 438 \mathrm{~cm}^{-1}[38,39]$. The absorption bands appeared around $\sim 3846, \sim 3736, \sim 2923$ and $\sim 2854$ represent characteristic vibrations of aliphatic $\mathrm{C}-\mathrm{H}$ groups [39]. The wide band at $3428 \mathrm{~cm}^{-1}$ can be attributed to the $\mathrm{O}-\mathrm{H}$ stretching vibrations [38] and the weak band at $1638 \mathrm{~cm}^{-1}$ is assigned to the $\mathrm{H}-\mathrm{O}-$ $\mathrm{H}$ bending vibrations [24] in hydroxyl groups which might be due to the employment of the $\mathrm{KBr}$ pellet technique as $\mathrm{KBr}$

Table 1. The crystallite size ' $D$ ', lattice parameter ' $a$ ', cut-off wavelength ( $\left.\lambda_{\text {cut-off }}\right)$, optical band gap $\left(E_{\mathrm{g}}\right)$ and Urbach energy $\left(E_{\mathrm{U}}\right)$ of pure and Co-substituted $\mathrm{NiO}$ nanoparticles.

\begin{tabular}{lccccc}
\hline Sample & $\begin{array}{c}\text { Average crystallite } \\
\text { size } D(\mathrm{~nm})\end{array}$ & $\begin{array}{c}\text { Lattice } \\
\text { parameter } a(\AA)\end{array}$ & $\begin{array}{c}\text { Cut-off wavelength } \\
\lambda_{\text {cut-off }}(\mathrm{nm})\end{array}$ & $\begin{array}{c}\text { Band gap } \\
E_{\mathrm{g}}(\mathrm{eV})\end{array}$ & $\begin{array}{c}\text { Urbach energy } \\
E_{\mathrm{U}}(\mathrm{eV})\end{array}$ \\
\hline $\mathrm{NiO}$ & 24 & 4.1742 & 317 & 3.91 & 0.34 \\
$\mathrm{Ni}_{0.9} \mathrm{Co}_{0.1} \mathrm{O}$ & 38 & 4.1760 & 361 & 3.89 & 0.38 \\
$\mathrm{Ni}_{0.8} \mathrm{Co}_{0.2} \mathrm{O}$ & 47 & 4.1778 & 370 & 3.66 & 1.04 \\
$\mathrm{Ni}_{0.6} \mathrm{Co}_{0.4} \mathrm{O}$ & 37 & 4.1778 & 425 & 3.10 & 2.58 \\
\hline
\end{tabular}




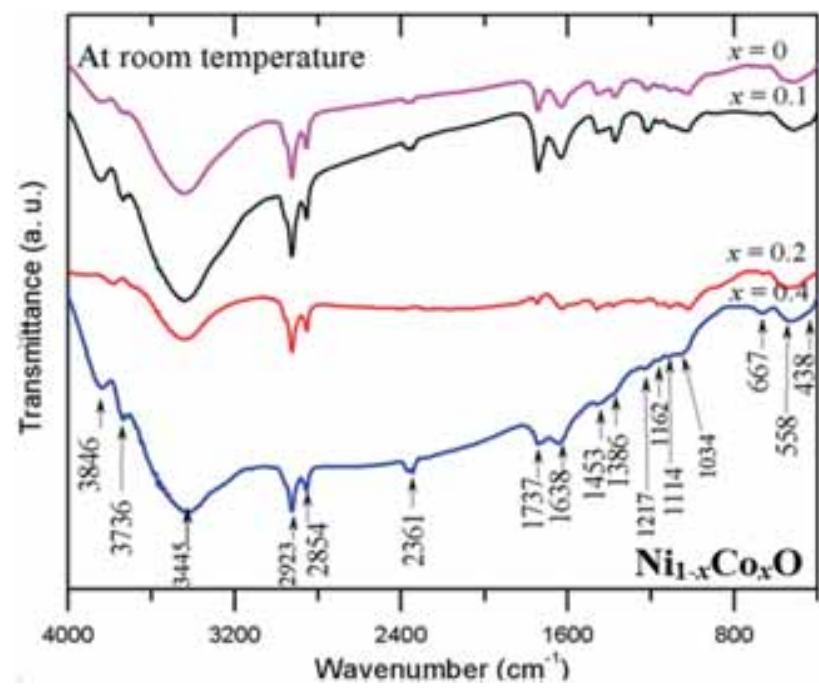

Figure 2. FTIR spectra of $\mathrm{Ni}_{1-x} \mathrm{Co}_{x} \mathrm{O}$ nanoparticles.

is highly hygroscopic. The bands at $\sim 2361$ and $\sim 1737 \mathrm{~cm}^{-1}$ are assigned to the stretching vibrations of $\mathrm{C}=\mathrm{O}$ due to the absorption of $\mathrm{CO}_{2}$ from the atmosphere [40-42]. The band at $\sim 1450 \mathrm{~cm}^{-1}$ contributes to $\mathrm{C}-\mathrm{H}$ scissoring [43]. Moving further, the band appeared at $1386 \mathrm{~cm}^{-1}$ is primarily due to the symmetric stretching vibrations of $\mathrm{O}-\mathrm{C}=\mathrm{O}$ groups. The peak at $1217 \mathrm{~cm}^{-1}$ may be assigned to vibrations on account of $\mathrm{C}-\mathrm{H}$ wagging. The next small kink at $\sim 1162 \mathrm{~cm}^{-1}$ can be attributed to the $\mathrm{C}-\mathrm{O}$ stretching vibrations. A faint shoulder at $1114 \mathrm{~cm}^{-1}$ may be assigned to in and out plane deformations in $\mathrm{C}-\mathrm{H}$ bonds. The band around $\sim 1034 \mathrm{~cm}^{-1}$ is due to the asymmetric stretching of the resonance interaction between vibration modes of oxide ions in the nanoparticles [41]. The vibrations of nickel species are usually active in the wavenumber range of $700-400 \mathrm{~cm}^{-1}$. A close observation of this region resulted in yielding two absorption bands at $\sim 667$ and $\sim 438 \mathrm{~cm}^{-1}$ and shoulder at around $\sim 558 \mathrm{~cm}^{-1}$ which can be easily deconvoluted to obtain Gaussian absorption bands. The first weak but well defined absorption band at $\sim 667 \mathrm{~cm}^{-1}$ can be attributed to the $\mathrm{Ni}-\mathrm{O}-\mathrm{H}$ vibrations due to the formation of nickel hydroxide units. The weak shoulder around $558 \mathrm{~cm}^{-1}$ may be ascribed to the bond bending vibrations of $\mathrm{Ni}-\mathrm{O}$ units whereas the last band at $\sim 438 \mathrm{~cm}^{-1}$ is due to the stretching vibrations of $\mathrm{Ni}-\mathrm{O}$ units. The position of stretching vibrations of Ni-O units confirmed the formation of nanoparticles [44]. The peak due to $\mathrm{Ni}-\mathrm{O}-\mathrm{H}$ vibrations exhibits a loss in intensity on the initial incorporation of cobalt in the $\mathrm{NiO}$ lattice indicating that the formation of nickel hydroxide units is less favourable but further addition of cobalt content leads to an enhancement in the intensity of this peak again. The $\mathrm{Ni}-\mathrm{O}$ peaks exhibit a very small red shift indicating a vibrational distortion due to the presence of cobalt species in $\mathrm{NiO}$ units as the atomic mass of cobalt is higher than that of nickel [45].

\subsection{SEM-EDS and TEM}

In order to obtain insight information of morphology, distribution and shape of the prepared nanoparticles, SEM is performed. Figure $3 \mathrm{a}-\mathrm{d}$ show the morphology of $\mathrm{Ni}_{1-x} \mathrm{Co}_{x} \mathrm{O}$ $(x=0,0.1,0.2$ and 0.4$)$ nanoparticles annealed at $400^{\circ} \mathrm{C}$. Inspection of these images reveals that particles are nonspherical in shape and are agglomerated. The agglomeration is due to the nano-dimension of particles [35]. The addition of $\mathrm{Co}^{2+}$ ions improves the morphology with suitable concentration but as the concentration is increased from $x=0.2$ to 0.4 , particles aggregate. We can say that insertion of cobalt ions leads to a change in the shape as compared to pure $\mathrm{NiO}$ nanoparticles. Thus the morphology can be tuned by varying the amounts of doping into the lattice. Qualitative elemental composition was measured by EDS. The EDS spectra as shown in figure 4 clearly validate that the synthesized nanoparticles are made up of $\mathrm{Ni}, \mathrm{O}$ and $\mathrm{Co}$ ions only. The presence of the peak corresponding to carbon may be due to the use of grid during analysis [40]. No other peaks corresponding to any type of salt impurity are present which confirms the high purity of the synthesized nanoparticles. Figure 5 depicts the TEM images of $\mathrm{Ni}_{1-x} \mathrm{Co}_{x} \mathrm{O}(x=0,0.1,0.2$ and 0.4$)$ nanoparticles with different concentrations of cobalt. All the prepared samples show the non-spherical structure of the synthesized nanoparticles.

\subsection{UV-Vis spectra}

The optical absorption spectra of $\mathrm{Ni}_{1-x} \mathrm{Co}_{x} \mathrm{O}(x=0,0.1,0.2$ and 0.4 ) nanoparticles are recorded by using double distilled water as a solvent and are shown in figure 6a. The absorption edge of these spectra can be used to obtain the band gap of nanoparticles because the absorption edge arises due to the electronic transitions from the valence band (VB) to the conduction band (CB) [46]. The cut-off wavelength $\left(\lambda_{\text {cut-off }}\right)$ has been evaluated by $x$-axis intercept of the absorption edge and the obtained values are reported in table 1 . A small red shift in $\lambda_{\text {cut-off }}$ is evident from table 1 for cobalt substituted nanoparticles in comparison with the pure $\mathrm{NiO}$ nanoparticles. This shift indicates a decrease in the band gap as $\lambda_{\text {cut-off }}$ usually exhibits an inverse relation with the optical band gap. The band gap is an important factor to decide the photocatalytic activity of nanomaterials [47]. The optical band gap $E_{\mathrm{g}}$ can be calculated using the Tauc's method, by extrapolating the linear portion of the curve to the zero absorption edge [48]:

$$
(\alpha h v)=B\left(h v-E_{\mathrm{g}}\right)^{n} .
$$

Here $\alpha$ is the absorption coefficient, $h v$ is the energy of photon, $E_{\mathrm{g}}$ is the optical band gap, $B$ is the material constant, $n$ is $1 / 3,1 / 2,2$ and 3 for direct forbidden, direct allowed, indirect allowed and indirect forbidden transitions, respectively [46]. $\mathrm{NiO}$ is considered to be a material with a direct band gap energy, hence $n=1 / 2$ for direct allowed transitions [48]. The respective Tauc's plot is shown in figure $6 \mathrm{~b}$ and the 


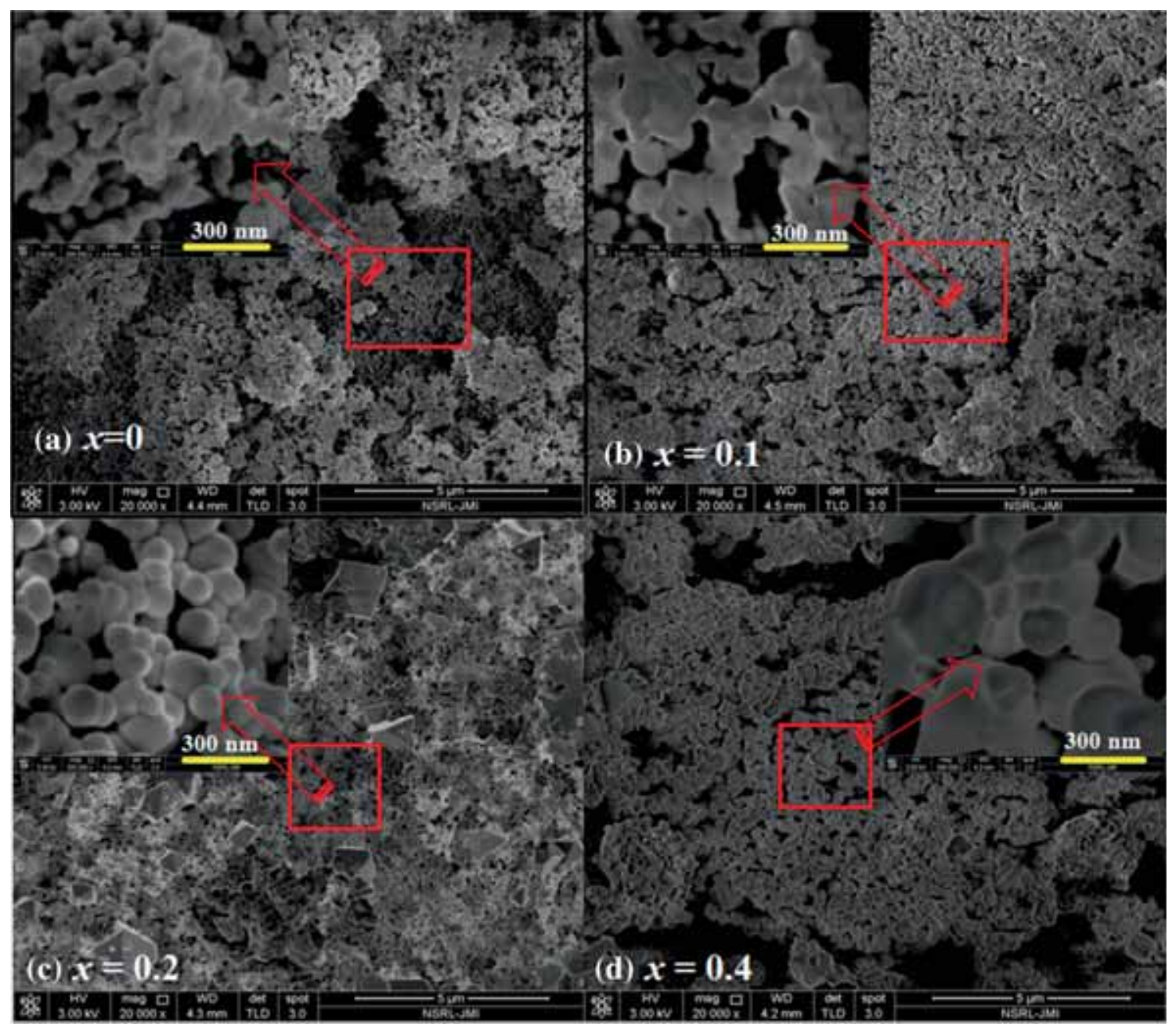

Figure 3. $\mathrm{SEM}$ images of $\mathrm{Ni}_{1-x} \mathrm{Co}_{x} \mathrm{O}$ nanoparticles (the inset shows the magnified image of the selected area).

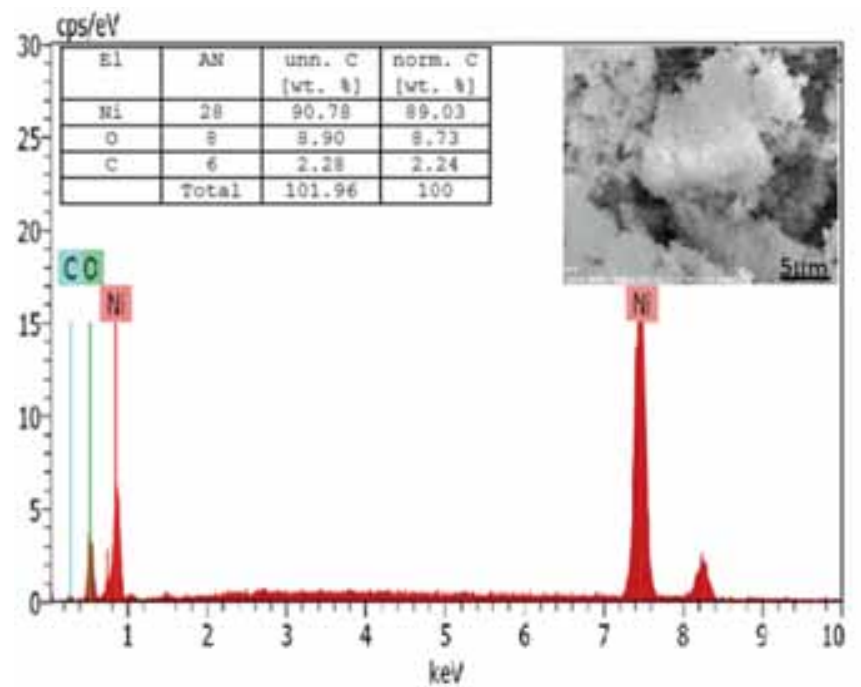

(a)

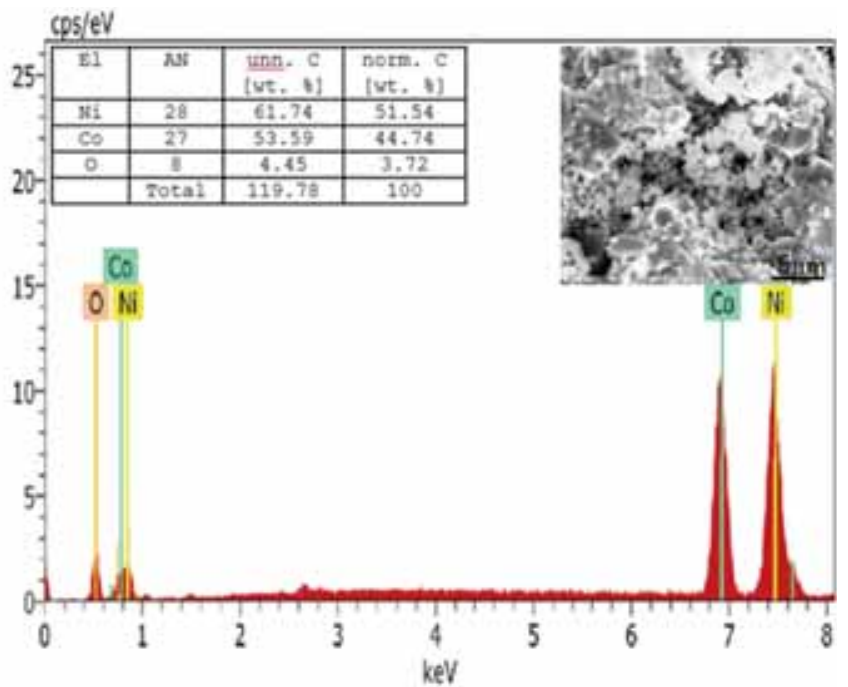

(b)

Figure 4. EDS spectra of $\mathrm{Ni}_{1-x} \mathrm{Co}_{x} \mathrm{O}$ for (a) $x=0$ and (b) $x=0.4$ (the inset shows the image of the area selected for EDS). 


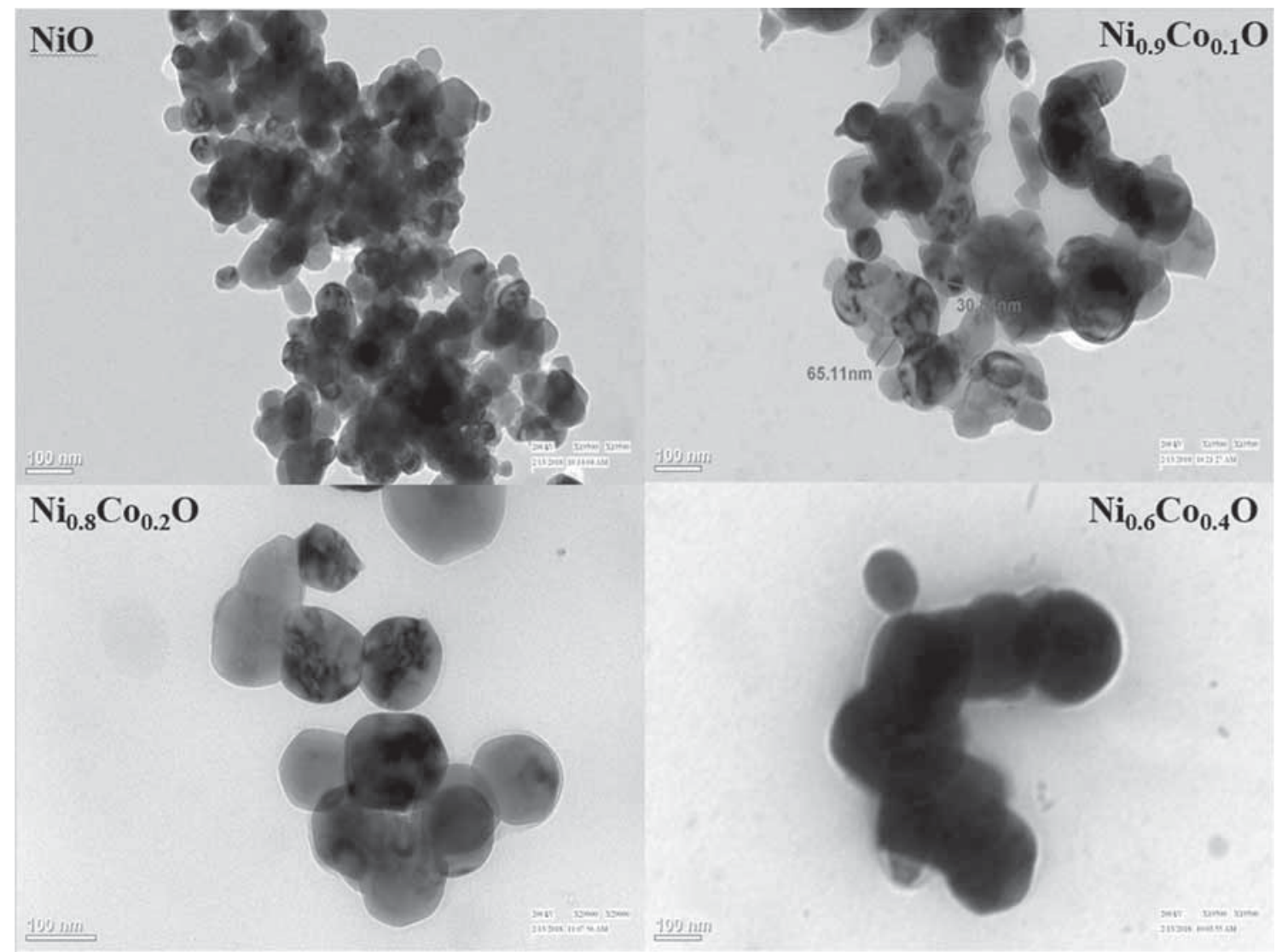

Figure 5. TEM images of pure and cobalt substituted $\mathrm{NiO}$ nanoparticles.
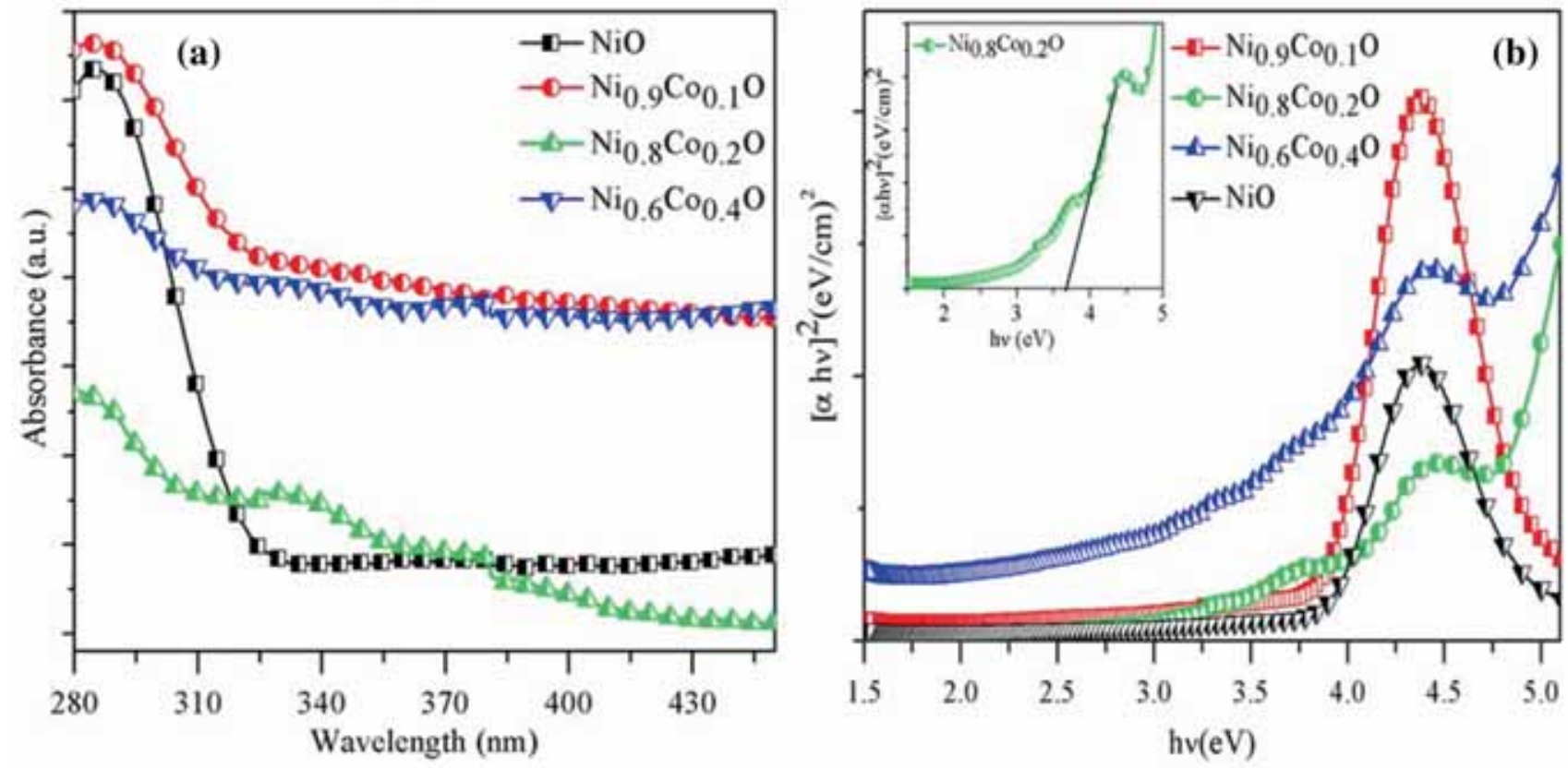

Figure 6. (a) Absorbance spectra of $\mathrm{Ni}_{1-x} \mathrm{Co}_{x} \mathrm{O}$ nanoparticles and (b) Tauc's plot for $\mathrm{Ni}_{1-x} \mathrm{Co}_{x} \mathrm{O}$ nanoparticles. 


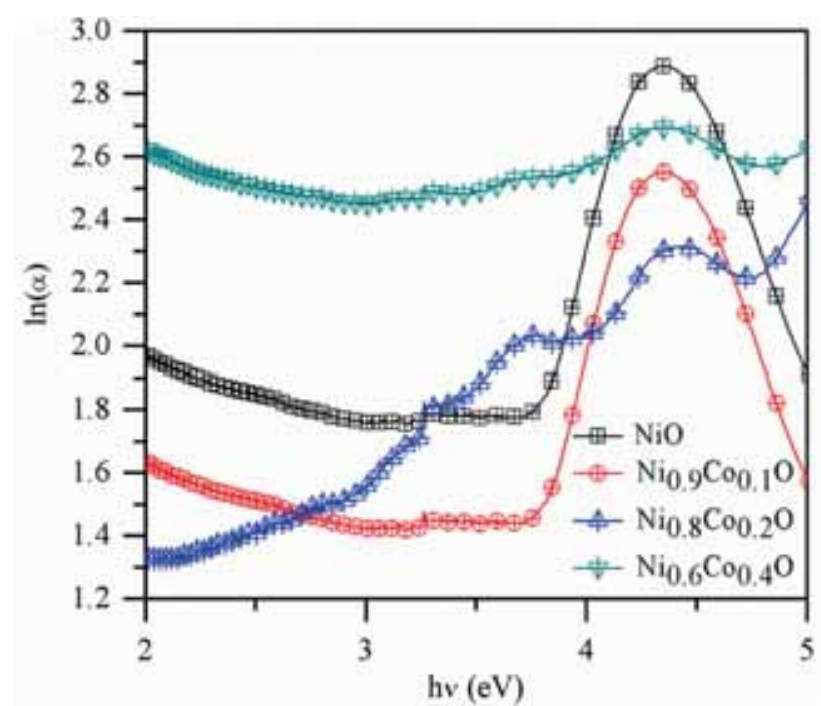

Figure 7. Urbach plot of $\mathrm{Ni}_{1-x} \mathrm{Co}_{x} \mathrm{O}$ nanoparticles.

obtained values of the band gap are listed in table 1. As can be seen, the optical band gap for pure $\mathrm{NiO}$ is $3.91 \mathrm{eV}$ which is in agreement with the literature [38] and it is decreasing with an increase in cobalt concentration. These compositional trends in $E_{\mathrm{g}}$ can be understood from the corresponding compositional variations in the crystallite size $(D)$ and due to the incorporation of cobalt. A little variation in $D$ usually leads to marked broadening of the absorption edge which might be due to the fact that the lower crystallite size leads to higher number of defects whereby creating additional energy levels of defects. Therefore, a part of energy is absorbed by the photon and is transferred to excite it in the energy level of the defect instead of the CB, which ultimately leads to an increase in the optical band gap. Moreover, the optical band gap of cobalt oxide is usually less than that of $\mathrm{NiO}$ which might force the composition containing the highest cobalt content to exhibit a synergic effect between the crystallite size and cobalt substitution. These results are firmly corroborated by the corresponding compositional trends in $\lambda_{\text {cut-off }}$.

The band tail energy or Urbach energy $\left(E_{\mathrm{U}}\right)$, which characterizes the width of the localized states, is calculated using the formula [48]:

$$
\alpha=\alpha_{0} \exp \left(\frac{h v}{E_{\mathrm{U}}}\right)
$$

where $\alpha_{0}$ is the constant. The above equation can be utilized to draw the Urbach plot $(\ln \alpha v s$. $h v)$. Figure 7 shows the Urbach plots of the prepared nanoparticles. The Urbach energy is evaluated from reciprocal of the slope of the linear portion of the absorption edge to study the disorder for all compositions and the obtained values are listed in table 1 . One can clearly observe from table 1 that compositional trends in $E_{\mathrm{U}}$ are in synchronization with the cut-off wavelength which

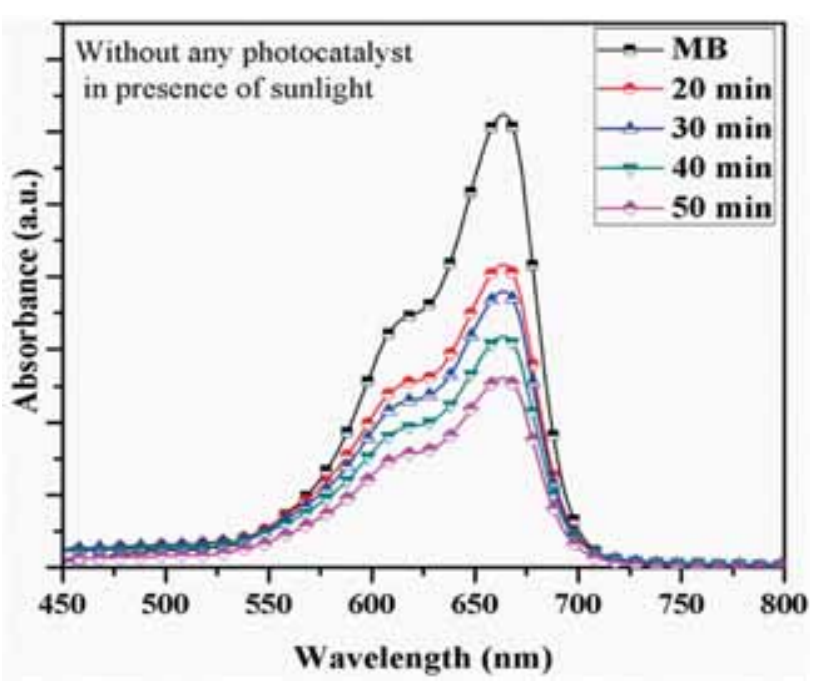

Figure 8. UV-Vis spectroscopic variation of $\mathrm{MB}$ dye solution without any photocatalysts.

validates the effect of Co incorporation in the $\mathrm{NiO}$ structure as concluded from $E_{\mathrm{g}}$.

\subsection{Photocatalytic activity of $\mathrm{Ni}_{1-x} \mathrm{Co}_{x} \mathrm{O}$ nanoparticles}

In the absence of pure $\mathrm{NiO}$ and $\mathrm{Co}$-doped $\mathrm{NiO}$ photocatalysts, the sunlight photolysis of aqueous MB dye solution was tested. It was found that MB dye cannot be easily degraded by sunlight photolysis shown in figure 8 . The typical absorption spectra of $\mathrm{MB}$ under solar light radiation in the presence of $\mathrm{Ni}_{0.9} \mathrm{Co}_{0.1} \mathrm{O}$ and $\mathrm{Ni}_{0.6} \mathrm{Co}_{0.4} \mathrm{O}$ nanoparticles are shown in figure 9. It can be clearly observed that the MB absorption band at $664 \mathrm{~nm}$ decreases gradually with time and almost flattens after $50 \mathrm{~min}$ of irradiation time indicating the photodegradation of MB (see the inset in figure 9). The percentage degradation as function of time was calculated for each photodegradation by considering the maximum absorbance at $664 \mathrm{~nm}$ as the reference. The reaction kinetics is determined by plotting $\ln C_{0} / C_{\mathrm{t}} v s$. irradiation time $(t)$ for the photocatalytic decolourization process. Figure 10 typically shows the effect of cobalt concentration doped in $\mathrm{NiO}$ nanoparticles at a fixed concentration of cobalt ions $(x=0.1$ and 0.4$)$ on photocatalytic decolourization kinetics of MB dye solution. Reaction kinetics gives enough information about the reaction rates. The result showed that the photocatalytic decolourization of MB dye obeys apparently pseudo-first order kinetics and the rate expression is given by [4]:

$$
\ln \frac{C_{0}}{C_{\mathrm{t}}}=k t
$$

where $C_{0}$ is the initial concentration of $\mathrm{MB}$ dye, $C_{\mathrm{t}}$ is the concentration at time $t$ and $k$ is the pseudo-first order rate constant. The rate constant, $k$ was found to be 0.044 and $0.059 \mathrm{~min}^{-1}$ in the case of $\mathrm{Ni}_{0.9} \mathrm{Co}_{0.1} \mathrm{O}$ and $\mathrm{Ni}_{0.6} \mathrm{Co}_{0.4} \mathrm{O}$ nanoparticles, 


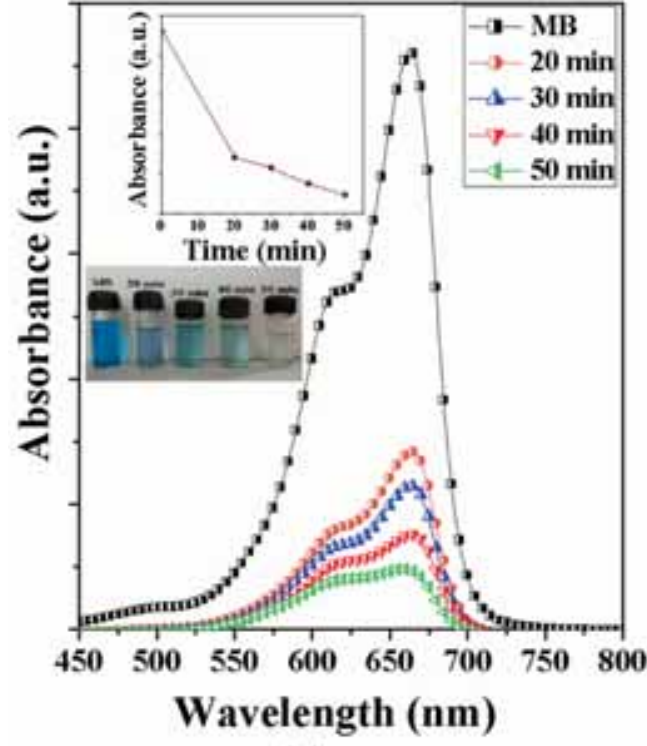

(a)

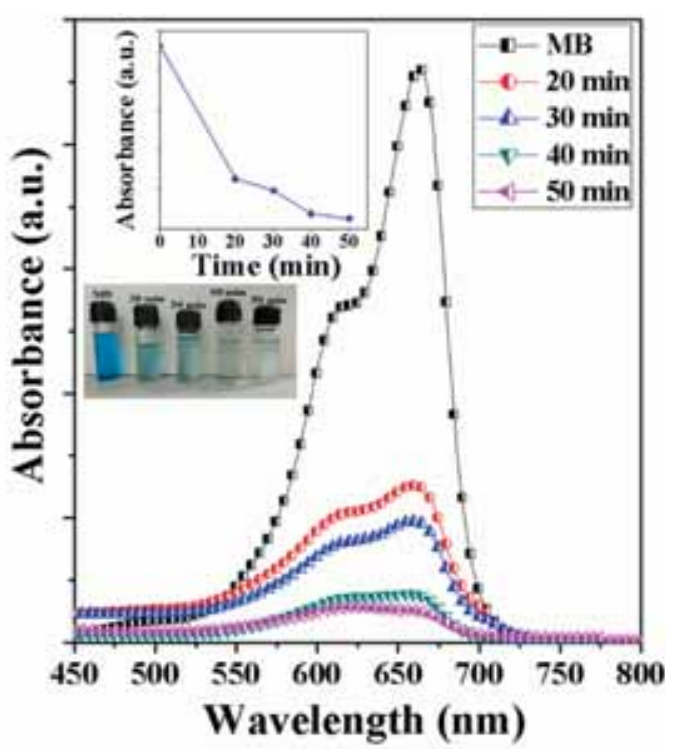

(b)

Figure 9. UV-Vis spectroscopic variation of $\mathrm{MB}$ dye solution in the presence of (a) $\mathrm{Ni}_{0.9} \mathrm{Co}_{0.1} \mathrm{O}$ and (b) $\mathrm{Ni}_{0.6} \mathrm{Co}_{0.4} \mathrm{O}$ nanoparticles.

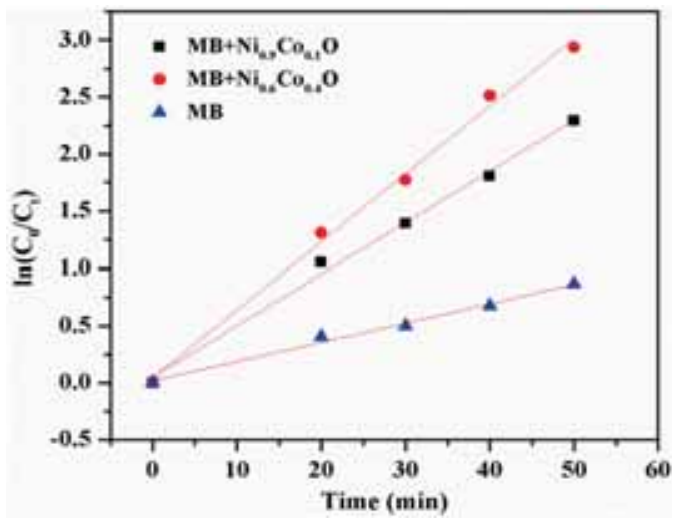

Figure 10. Variation of $\mathrm{MB}$ concentration $\ln C_{0} / C$ with time over $\mathrm{Ni}_{0.9} \mathrm{Co}_{0.1} \mathrm{O}$ and $\mathrm{Ni}_{0.6} \mathrm{Co}_{0.4} \mathrm{O}$ nanoparticles as catalysts. respectively. The obtained correlation coefficient $\left(R^{2}\right)$ is 0.976 and 0.979 for $\mathrm{Ni}_{0.9} \mathrm{Co}_{0.1} \mathrm{O}$ and $\mathrm{Ni}_{0.6} \mathrm{Co}_{0.4} \mathrm{O}$ nanoparticles, respectively. While without any photocatalysts, the rate constant and correlation coefficient were found to be $0.0168 \mathrm{~min}^{-1}$ and 0.988 . It was found that higher cobalt ion content results in an enhanced degradation rate. This is because cobalt substitution has a direct influence on the optical band gap of $\mathrm{NiO}$ nanoparticles promoting the photocatalytic activity. An in-depth review of the literature has been carried out to find decolourization of MB using different materials as photocatalysts and the typical comparison with the present study is reported in table 2. As can be seen, the materials synthesized in present work are performing well towards the photocatalytic degradation of MB. Figure 11 shows the degradation efficiencies for cobalt substituted $\mathrm{NiO}$

Table 2. A comparison with the literature for decolourization of MB.

\begin{tabular}{lcccc}
\hline Photocatalyst material & Light source & Time (min) & \% Degradation & References \\
\hline Mn doped NiO nanoparticles & UV light & 210 & 92 & {$[3]$} \\
$\mathrm{TiO}_{2}$ & UV light & 250 & 100 & {$[6]$} \\
$\mathrm{NiO}-\mathrm{ZnO}$ nanocomposite & UV light & 80 & 72 & {$[52]$} \\
$\mathrm{ZnO}$ nanoparticles & UV light & 120 & 90 & {$[53]$} \\
$\mathrm{NiO}$ nanoparticles & Sunlight & 120 & 94 & {$[54]$} \\
$\mathrm{ZnO}$ nanoparticles & UV light & 60 & $>90$ & {$[55]$} \\
$\mathrm{WO}_{3}-\mathrm{GO}$ nanocomposite & Sunlight & 70 & 94 & {$[42]$} \\
$\mathrm{Au}_{\mathrm{ZnO}-\mathrm{CeO}}$ nanohybrid & N. M. & 8 & 100 & {$[56]$} \\
$\mathrm{Ni}_{0.9} \mathrm{Co}_{0.1} \mathrm{O}$ & Sunlight & 50 & $\sim 89$ & $\mathrm{~T}$. W. \\
$\mathrm{Ni}_{0.6} \mathrm{Co}_{0.4} \mathrm{O}$ & Sunlight & 50 & $\sim 94$ & $\mathrm{~T}$. W. \\
\hline
\end{tabular}

T. W. $\rightarrow$ This work 


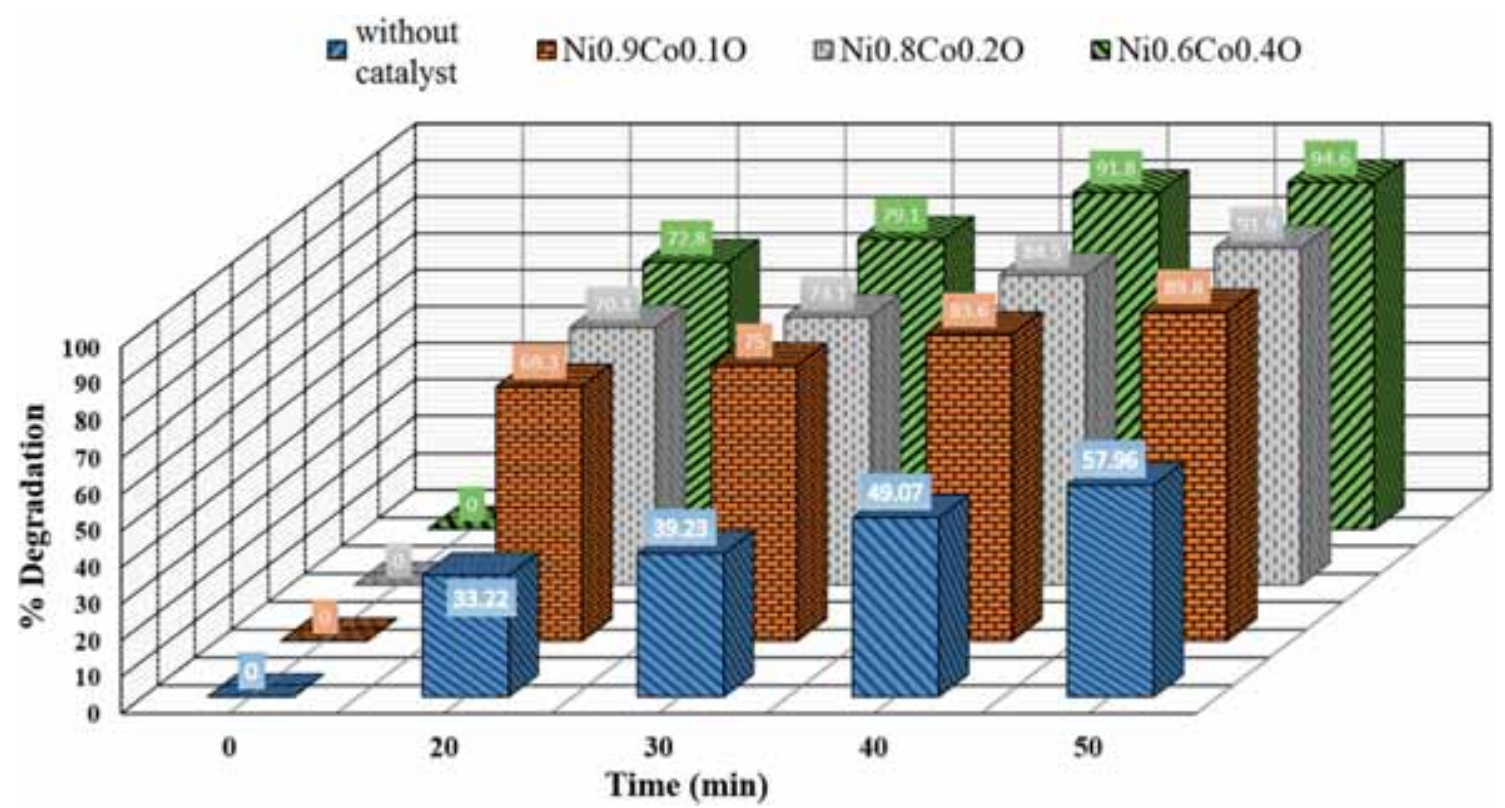

Figure 11. Percentage degradation of $\mathrm{MB}$ dye solution as a function of irradiation time (min) in the presence of nanoparticles as catalysts.

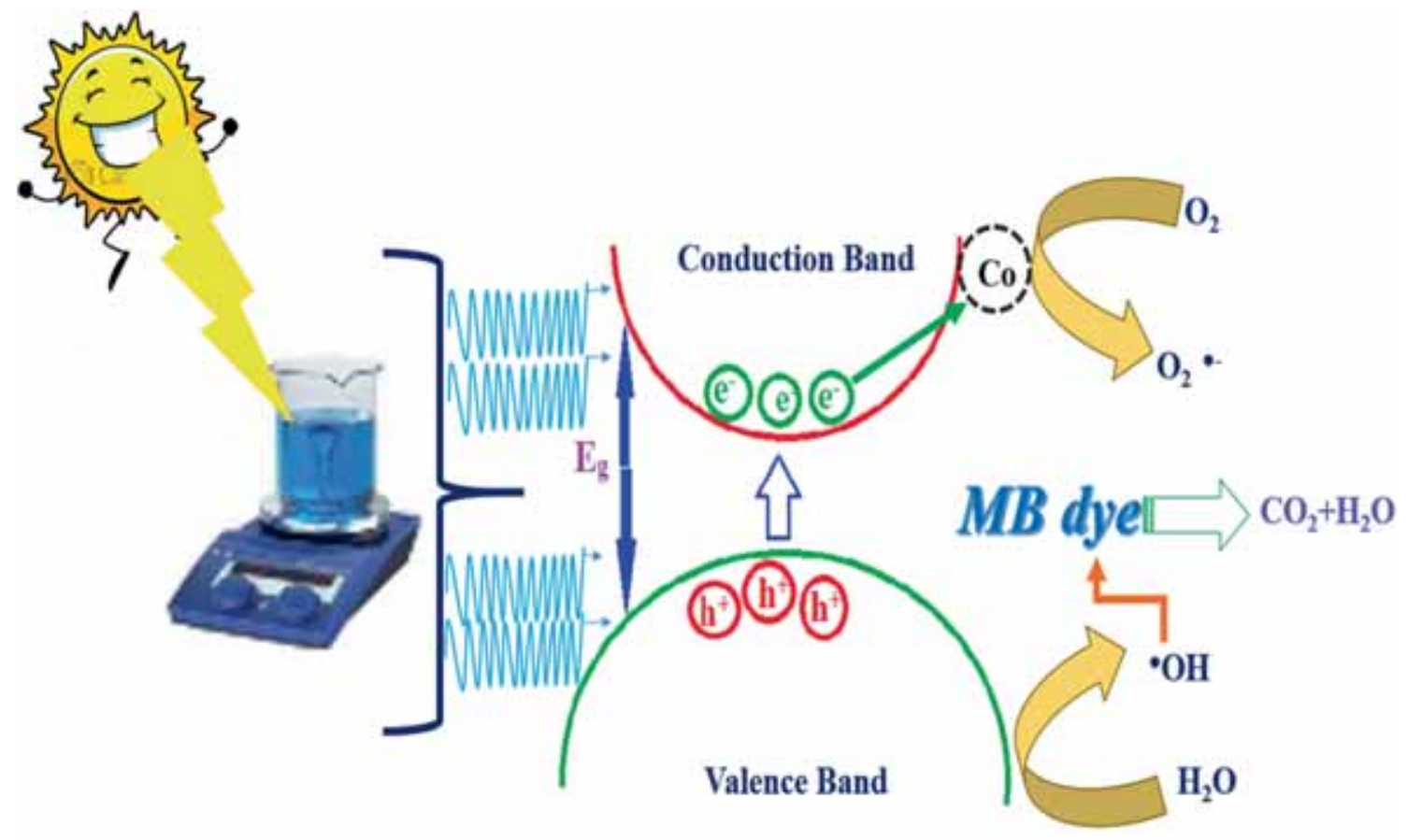

$\mathrm{NiO}$

Figure 12. Mechanism for the dye degradation process in the presence of the catalyst.

nanoparticles as catalysts. It is clearly evident that better degradation is achieved in the presence of $\mathrm{Ni}_{0.6} \mathrm{Co}_{0.4} \mathrm{O}$ indicating that the doping of cobalt ions enhances the photocatalytic activity of NiO nanoparticles. From the optical properties, it can be inferred that there is a decrease in the band gap on increasing the concentration of cobalt doping. As sunlight radiation is used to excite the nanoparticles, the photocatalytic activity increases with the decrease in the optical band gap [50]. As the band gap is decreased, generation of the $\mathrm{e}^{-} / \mathrm{h}^{+}$pair is enhanced, thus the photocatalytic process is 
accelerated. The photodegradation mechanism of MB dye solution using $\mathrm{Ni}_{1-x} \mathrm{Co}_{x} \mathrm{O}$ nanoparticles is shown in figure 12 which can be deduced as: when $\mathrm{Ni}_{1-x} \mathrm{Co}_{x} \mathrm{O}$ nanoparticles are exposed to sunlight, the electrons present in the VB acquire enough energy so as to get excited to the CB leaving holes behind in the VB. These holes and electrons are now available for the oxidation and reduction processes, respectively. The electrons present in the $\mathrm{CB}$ are transferred to adsorbed oxygen $\left(\mathrm{O}_{2}\right)$ on the surface of nanoparticles to give oxygen $\left(\mathrm{O}^{2 \bullet-}\right)$ radicals. The photocatalytic performance of nanoparticles as evident from figure 10 increases with an increase in the substitution of cobalt. It may be due to the fact that doping of cobalt in $\mathrm{NiO}$ accelerates the transfer of the electron in the $\mathrm{CB}$ to the dissolved oxygen. As a result of this, chances of recombination of electrons-holes get reduced leading to advancement in the photocatalysis process. The photogenerated holes will abstract electrons from $\mathrm{H}_{2} \mathrm{O}$ to produce hydroxyl $\left({ }^{\bullet} \mathrm{OH}\right) \mathrm{rad}-$ icals. The hydroxyl radical is an efficient oxidant for the partial or complete degradation of organic chemicals [51]. These hydroxyl radicals facilitate the oxidation of $\mathrm{MB}$ dye molecules adsorbed on the surface of nanoparticles leading to the decolourization of MB dye.

\section{Conclusion}

The pure $(x=0)$ and cobalt substituted ( $x=0.1,0.2$ and $0.4) \mathrm{Ni}_{1-x} \mathrm{Co}_{x} \mathrm{O}$ nanoparticles were synthesized successfully by the chemical co-precipitation method. The XRD analysis revealed the FCC structure of the prepared nanoparticles. The presence of all functional groups was observed by FTIR analysis. The SEM micrographs affirm the non-spherical morphology of the synthesized nanoparticles. Compositional analysis (EDS) ascertained the existence of $\mathrm{Ni}, \mathrm{O}$ and $\mathrm{Co}$ in the synthesized nanoparticles. The purity of nanoparticles is confirmed by the absence of impurity peaks in EDS spectra. TEM images showed an irregular shape of nanoparticles with different nanosizes. The analysis of the optical properties using UV-Vis spectroscopy indicated a decrease in the optical band gap with an enhanced substitution of cobalt ions. The photocatalytic activity of the synthesized nanoparticles under solar light irradiation revealed that $\mathrm{MB}$ dye can be almost degraded in $50 \mathrm{~min}$ in the presence of the $\mathrm{Ni}_{0.6} \mathrm{Co}_{0.4} \mathrm{O}$ sample.

\section{References}

[1] Szyula A, Guibal E, Ruiz M and Sastre A M 2008 Colloids Surf. A 330219

[2] Purkait M K, Dasgupta S and De S 2004 Sep. Purif. Technol. 3781

[3] Sankara S, Sharma S K, An N, Lee H, Kim D Y, Im Y B et al 2016 Optik 12710727

[4] Malik R, Rana P S, Tomer V K, Chaudhary V, Nehra S P and Duhan S 2016 Micro. Mes. Mater. 225245
[5] Swaminathan M, Muruganandha M and Sillanpaa M 2013 Int. J. Photoenergy 2013683682

[6] Shrivastava V S 2012 Arch. Appl. Sci. Res. 41244

[7] Song S, Fan J, Zhan L, Liu Z, Chen J and Xu X 2010 Electrochim. Acta $\mathbf{5 5} 3606$

[8] Rauf M A, Meetani M A, Khaleel A and Ahmed A 2010 Chem. Eng. J. 157373

[9] Lee K M, Lai C W, Ngai K S and Juan J C 2016 Water Res. 88 428

[10] Gupta S M and Tripathi M 2011 Chin. Sci. Bull. 561639

[11] Desouky O A, Khalil M M H and Kattab H A 2013 IJSR 3316

[12] Wang C, Cui X, Liu J, Zhou X, Cheng X, Sun P et al 2016 ACS Sens. 1131

[13] Dirksen J A, Duval K and Ring T A 2001 Sens. Actutators B 80106

[14] Park J Y, Choi S W, Jung S H and Kim S S 2012 J. Nanosci. Nanotechnol. 21288

[15] Hotovy I, Huran J, Spiess L, Hascik S and Rehacek V 1999 Sens. Actuators B 57147

[16] Park B and Cairns E J 2011 Electrochem. Commun. 1375

[17] Jiao Z, Wu M, Qin Z and Xu H 2003 Nanotechnol. 14458

[18] Browne M P, Nolan H, Berner N C, Dulsberg G S, Colavita P E and Lyons M E G 2016 Int. J. Electrochem. Sci. 116636

[19] Yan W and Ke J J 1996 Mater. Res. Bull. 151

[20] Santhoshkumar A, Kavitha H P and Suresh R 2016 J. Am. Chem. Soc. 2230

[21] Gondal M A, Saleh T A and Drmosh Q A 2012 Appl. Surf. Sci. 2582982

[22] Anandan K and Rajendran V 2011 Solid State Electron. 1443

[23] Qiao H, Wei Z, Yang H, Zhu L and Yan X 2009 J. Nanomater. 2009795928

[24] Alagiri M, Ponnusamy S and Muthamizhchelvan C $2012 \mathrm{~J}$. Mater. Sci. Mater. Electron. 23728

[25] Jeevanandam P and Pulimi V R R 2012 Indian J. Chem. A 51 586

[26] Mallick P and Mishra N C 2012 Am. J. Mater. Sci. 266

[27] Motahari F, Mozdianfard M R, Soofivand F and Niasari M S 2014 RSC Adv. 454

[28] Gnanasekaran L, Hemamalini R, Saravanan R, Ravichandran K, Gracia F, Agarwal S et al 2017 J. Photochem. Photobiol. B 17343

[29] Mallick P, Rath C, Biswal R and Mishra N C 2009 Indian J. Phys. 83517

[30] Tang C W, Wang C B and Chein S H 2008 Thermochim. Acta 47368

[31] Malik R, Tomer V K, Duhan S, Nehra S P and Rana P S 2015 Energy Environ. Focus 4340

[32] Suresh S and Podder J 2015 Int. J. Nanoparticles 8289

[33] Cullity B D 1956 Elements of X-ray diffraction (Greece: Addison-Welsey Publishing Company Reading Mass)

[34] Agrawal S, Parveen A and Azam A 2017 J. Luminiscence 184 250

[35] Ponnusamy P M, Agilan S, Muthukumarasamy N, Raja M and Velauthapillai D 2016 J. Mater. Sci.: Mater. Electron. 27 399

[36] Zhang Y, Zhou Q, Lv X, Ma Y, Zhong J and Sun X 2015 Electrochim. Acta $\mathbf{1 7 8} 590$

[37] Wang X W, Zheng D L, Zhang P Z, Wang X E, Zhu Q Q, Ma P F et al 2017 Chem. Phys. Lett. 667260

[38] Anandan K and Rajendran V 2012 J. Nanosci. Nanotechnol. 2 24 
[39] Davar F, Fereshteh Z and Niasari M S 2009 J. Alloys Compd. 476797

[40] Krishnakath R, Jayakumar G, Irudayaraj A A and Raj A D 2016 Mater. Today: Proc. 31370

[41] Gandhi V, Ganesan R, Syedahamed H H A and Thaiyan M 2014 J. Phys. Chem. C 1189715

[42] Ismail A A, Faisal M and Haddad A A 2018 J. Env. Sci. 66 328

[43] edited by Lopez L L M and Sanchez J M 1993 Advanced topics in materials science and engineering (New York: Plenum Press) http://www.springer.com/in/book/9781461362302

[44] Kemary M E, Nagy N and Mehasseb I E 2016 Mater. Sci. Semicond. Process. 161747

[45] Christy A A, Ozaki Y and Gregoriou V G 2001 Modern fourier transform infrared spectroscopy 1st edn. (Greece: Elesvier Science)

[46] Talebian N and Jafarinezhad F 2013 Ceram. Int. 398311

[47] Malik R, Chaudhary V, Rana P S, Tomer V K and Duhan S 2016 Energy Environ. Focus 535
[48] Yadav A, Khasa S, Dahiya M S, Dalal S, Hooda A and Agarwal A 2016 Phys. Chem. Glasses: Eur. J. Glass Sci. Technol. B 57 146

[49] Wan X, Yuan M, Long S and Lan S 2013 Appl. Surf. Sci. 277 40

[50] Nagaveni K, Hegde M S, Ravishankar N, Subbanna G N and Madras G 2004 Langmuir 202900

[51] Liu R, Huang Y, Xiao A and Liu H 2010 J. Alloys Compd. 503 103

[52] Rahman I A, Ayob M T M and Radiman S 2014 J. Nanotech. 2014212694

[53] Jang Y J, Simer C and Ohm T 2006 Mater. Res. Bull. 41 67

[54] Ramasami A K, Reddy M V and Balakrishna G R 2015 Mater. Sci. Semicond. Process. 40194

[55] Shen W, Li Z, Wang H, Liu Y, Guo Q and Zhang Y 2008 J. Hazard. Mater. 152172

[56] Veziroglu S, Kuru M, Ghori M Z, Dokan F K, Hinz A M, Strunskus T et al 2017 Mater. Lett. 209486 